\title{
Tracking the dynamics of power sources and sinks during the martensitic transformation of a $\mathrm{Cu}-\mathrm{Al}-\mathrm{Ni}$ single crystal.
}

Lucia lanniciello, ${ }^{1}$ Michela Romanini, ${ }^{2}$ Lluís Mañosa, ${ }^{2}$ Antoni Planes, ${ }^{2}$ Kurt Engelbrecht, ${ }^{1}$ and Eduard Vives ${ }^{2}$ a)

${ }^{1)}$ Department of Energy Conversion and Storage, Technical University of Denmark, Anker Engelunds Vej, Building 301,

2800 Kgs. Lyngby, Denmark.

${ }^{2)}$ Departament de Física de la Matèria Condensada, Facultat de Física. Universitat de Barcelona, Diagonal, 647,

E-08028 Barcelona, Catalonia.

(Dated: 20 April 2020)

We have tracked the dynamics of the martensitic transformation in a $\mathrm{Cu}-\mathrm{Al}-\mathrm{Ni}$ single crystal by means of acoustic emission and infrared imaging techniques. A Fourier equation-based post-processing of temperature maps has enabled us to reveal the inhomogeneous and discontinuous character of heat power sources and sinks during the transiton. A good correlation between the dynamics of thermal and mechanical energy release has been evidenced. It has also been shown that the merging of martensitic interfaces results in an enhanced heat absorption.

Cu-based shape memory alloys undergo a thermoelastic martensitic transformation from a high symmetry cubic phase to a lower symetry phase which can be induced either by changing temperature or by applying an external stress ${ }^{1}$. Associated with this martensitic transition there are many interesting thermo-mechanical properties such as shape memory, superelasticity, and giant elastocaloric effects which confer these alloys a high technological interest ${ }^{2}$. The martensitic transition proceeds by the nucleation and growth of martensitic domains which occurs in an inhomogeneous and discontinuous manner ${ }^{3}$. While an inhomogenous and discontinuous character may influence the performances of these alloys in diverse applications, characterization of such a behaviour with good enough temporal and spatial resolution is still challenging.

Images of the direct evolution of the strain field have been obtained by combining optical imaging with the Digital Image Correlation (DIC) method ${ }^{4}$ or the so called GRID method ${ }^{5,6}$ Sudden changes in the strain field give rise to elastic waves (which encompass a release of mechanical energy) which can be detected by acoustic emission (AE) techniques with unprecedented temporal resolution. AE has been widely used to monitor martensitic transitions in many materials ${ }^{7}$. It has been shown that the martensitic transition proceeds by an avalanche dynamics ${ }^{8}$, strongly influenced by the existence of disorder in the system. The use of several AE transducers has enabled a localization of the source of these avalanches ${ }^{9}$. On the other hand, Infrared (IR) imaging has also been used to measure temperature changes taking place in the sample during the martensitic transition ${ }^{4,10,11}$. This technique has become particularly useful in the study of shape memory materials for elastocaloric purposes. IR images reveal the inhomogenous temperature distribution within the sample, but these temperature maps do not provide a direct picture of the time and location were thermal energy (latent heat) is released or absorbed. However, by a suitable post-processing treatment of IR data based on the Fourier equation it is possible to build up maps of the latent heat exchange ${ }^{12,13}$.

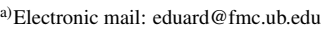

In this work we have coupled multi-transducer AE detection with IR imaging to study the mechanical and thermal energy exchange during the stress-induced martensitic transformation of a $\mathrm{Cu}-\mathrm{Al}-\mathrm{Ni}$ single crystal. These experiments have enabled us to track the dynamics of the martensitic transition by determining the time evolution of thermal and mechanical energy sources maps. While a global correlation between $\mathrm{AE}$ activity and latent heat exchange was established long ago ${ }^{14}$, IR and $\mathrm{AE}$ techniques reveal details on the microstructures formed during the martensitic transformation. The good correlation shown here at a microstructural level enables to gain insight on the dynamics of the martensitic transition. Some peculiarities found for the local release or absortion of latent heat may be useful in the potential use of shape memory alloys in cooling devices ${ }^{15}$.

We have studied a $\mathrm{Cu}-\mathrm{Al}-\mathrm{Ni}$ single crystalline wire produced by Nimesis Technology with nominal composition $\mathrm{Cu}_{70.6 \%} \mathrm{Al}_{25.7 \%} \mathrm{Ni}_{3.7 \%}$ atomic percent. The initial sample dimensions are $1 \mathrm{~mm}$ diameter and $\sim 30 \mathrm{~mm}$ length. According to the manufacturer the wire symmetry axis is oriented along the $\langle 100\rangle$ crystallographic direction, and the sample transforms from a high temperature cubic phase to a low temperature $18 \mathrm{R}$ martensitic phase. From DSC experiments (see Fig. 1(a)) performed on a small sample $(m=22.43 \mathrm{mg})$ we found the transition temperatures $M_{s}=250 \pm 1 \mathrm{~K}, M_{f}=$ $224 \pm 3 \mathrm{~K}, A_{s}=225 \pm 3 \mathrm{~K}$ and $A_{f}=285 \pm 3 \mathrm{~K}$, and latent heat $\Delta H=5.3 \pm 0.2 \mathrm{~J} / \mathrm{g}$. Stress-strain experiments at room temperature (see Fig. 1(b)) result in a transition stress of $\sim 110$ $\mathrm{MPa}$, and a transition strain $\sim 9 \%$. A schematic representation of the experimental setup is shown in Fig.1(c). The wire is tightly gripped to a Zwick/Roell Z005 testing machine. The resolution of the load cell is $0.01 \mathrm{~N}$ and the resolution in the crosshead extension $0.01 \mathrm{~mm}$. The initial length of the wire between grips (gauge length) is $L=24 \mathrm{~mm}$. After thermal equilibration at room temperature $\left(T_{0} \simeq 299 \mathrm{~K}\right)$, the sample has been loaded across the MT at a speed $0.1 \mathrm{~mm} / \mathrm{s}$, until reaching a maximum elongation of $2.3 \mathrm{~mm}$. After waiting 10 $\mathrm{s}$ for equilibration the sample has been unloaded at the same speed.

Two micro-80 AE transducers are acoustically coupled to the grips, as shown in Fig.1(c) (CH1 in the upper grip and $\mathrm{CH} 2$ in the lower grip). The signals from the transducers are 

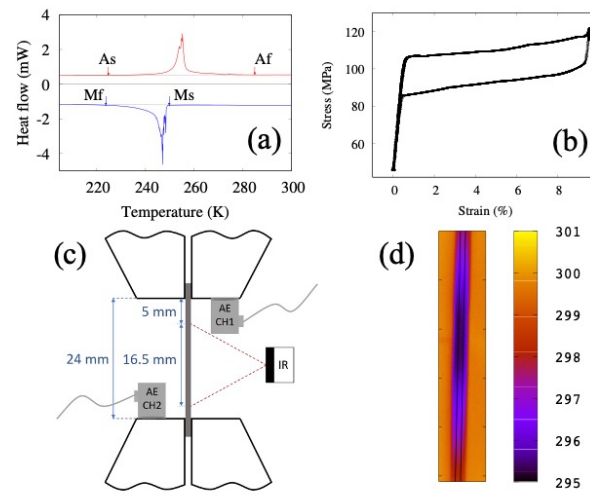

(d)



FIG. 1. (a) Calorimetric response of a small piece of the $\mathrm{Cu}-\mathrm{Al}-\mathrm{Ni}$ sample, (b) Stress-strain loop of the studied $\mathrm{Cu}-\mathrm{Al}-\mathrm{Ni}$ wire obtained at $T_{0}=299 \mathrm{~K}$. (c) Schematic of the experimental setup, indicating the sample dimensions and the position of the IR imaged region. (d) Example of IR image, corresponding to $t=54 \mathrm{~s}$ (frame 2070) during unloading. The diameter of the wire corresponds to 31 pixels. The observed inclination is due to the tilted orientation of the IR camera. The two black lines indicate the central zone of the wire (11 pixels width), where horizontal averages have been computed in order to obtain the vertical temperature profiles $T(z)$, as well as total averages in order to obtain the sample average temperature.

amplified $(60 \mathrm{~dB})$ and input into a PCI-2 acquisition system from Mistras Group working at $40 \mathrm{MHz}$. AE hits in every channel are determined as follows: when the voltage (in absolute value) exceeds a threshold $(25 \mathrm{~dB})$, the hit starts. When the voltage remains below threshold for more than $100 \mu \mathrm{s}$, the hit ends. From the list of detected hits, one can define located $\mathrm{AE}$ events when two consecutive hits, one on each channel, occur at times $t_{1}$ and $t_{2}$ (the subindex stands for the channel), with $\delta t=t_{1}-t_{2}\left(|\delta t|<\Delta_{\max }\right)$. The position of the event in the vertical direction $(z)$ can be computed as $z=\frac{L}{2}\left(1+\frac{\delta t}{\Delta_{\max }}\right)$. The value $\Delta_{\max }=14.2 \mu \mathrm{s}$ has been determined by a previous calibration. The source amplitude is determined by averaging the amplitudes of the hits in each channel.

A segment of the sample (see Fig.1(c)) is imaged by means of an infrared thermographic camera (InfraTec 8300) with a spatial resolution of $0.03226 \mathrm{~mm} / \mathrm{pixel}$ and a temperature resolution of $1 \mathrm{mK}$. A typical IR temperature map is shown in Fig.1(d). Despite all the efforts in avoiding radiation reflections, in improving the homogeneity in the sample emissivity and after carefully calibrating the sensor, a numerical smoothing treatment is still needed. The recorded frames have firs been corrected for few erroneous pixels that give temperatures out of any reasonable range. Data in these pixels are replaced by the average of the neighbouring pixels. After selecting the tilted rectangle that corresponds to the center of the wire (see the two black lines in Fig. 1(d)), we have performed horizontal averages (over 11 pixels) in order to obtain a collection of vertical profiles $T(i, k)$ with $i$ indicating the pixel and $k$ the frame number. The corresponding discretizations in the vertical direction and time are $\Delta z=0.03226 \mathrm{~mm}$ and $\Delta t=0.01$ s.

The time evolution of the measured force and the sample average temperature is plotted in Fig. 2(a). On loading the force shows a plateau which corresponds to the MT, during which the temperature of the sample increases by $3.36 \mathrm{~K}$. Upon unloading, the force also shows a plateau (at a lower value due to hystheresis) with an average temperature decrease of $-3.74 \mathrm{~K}$. In the bottom panel of Fig.2, AE activity (number of events located in the imaged region, per unit time) is shown as a function of time (black line). For both, loading and unloading runs, AE activity is maximum at the beginning and at end of the transition plateau. Red and blue curves in that figure correspond to the evolution of the total power source and sink, that will be discussed later.

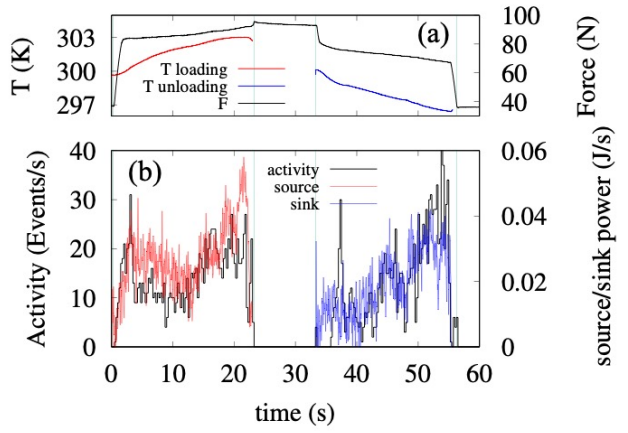

FIG. 2. Panel (a) displays the behaviour of the applied force (right scale) and the average sample temperature (left scale). The vertical lines indicate the loading regime, at $0.1 \mathrm{~mm} / \mathrm{s}$ for $23 \mathrm{~s}$, a pause of 10 $\mathrm{s}$ for thermal equilibration, and the unloading regime at $-0.1 \mathrm{~mm} / \mathrm{s}$ for $23 \mathrm{~s}$. Panel (b) shows the recorded acoustic activity (in bins of $0.25 \mathrm{~s}$ ), and the behaviour of the total heat power source and sink, computed every $10 \mathrm{~ms}$.

The location of the AE events along the vertical $z$ axis is shown as a function of time in Figures 3(a) (loading) and 3(b) unloading where the event amplitude is indicated by the colour code. The total number of located events during loading is 1713 (4775 hits in $\mathrm{CH} 1$ and 4221 hits in $\mathrm{CH} 2$ ) and during unloading is 1633 (8749 hits in $\mathrm{CH} 1$ and 6839 hits in $\mathrm{CH} 2$ ). During loading the activity concentrates in a single front that advances along the sample from bottom to top. This behaviour agrees with the fact that martensite nucleates in the bottom part of the sample and advances towards the austenitic upper part. When unloading, a first austenitic front advances from top to bottom but at a certain moment a second austenite-martensite front starts advancing upwards from the bottom. This occurs after a clear pause of activity of the top front, that was probably pinned by some defects. This coexistence of multiple martensitic fronts is related to the fact 
that the transformation is driven by strain contro ${ }^{16}$. Later, both fronts advance simultaneously and they merge close to the center of the sample.

It is worth noticing that inhomogeneous behaviour has been previously observed in other $\mathrm{Cu}$ based single crystalline samples under loading and unloading, using $\mathrm{AE}^{6,9}$, polarized-light optical microscopy ${ }^{16,17}$ and full-field strain measurements using an optical grid method ${ }^{6}$.

In general, the amplitude (and energy) of the events during unloading is higher than during loading. Interestingly, it is observed that the propagation of the front is not continuous but rather it exhibits intermittencies (as evidenced by the absences of hits for some time intervals, specially during unloading at $47 \mathrm{~s}$ and $49 \mathrm{~s}$ ). This finding reflects the well-known jerky behaviour of the martensitic transformation.

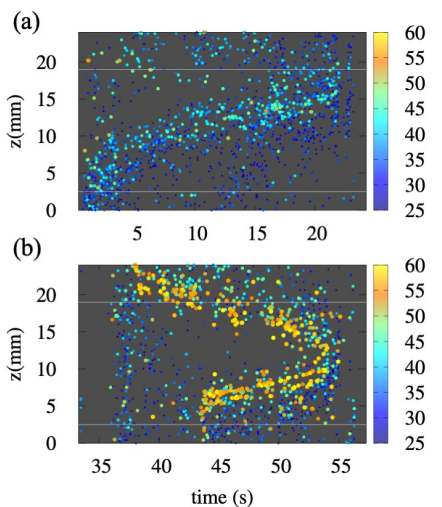

FIG. 3. Vertical position of detected AE events as a function of time for the loading (a) and unloading (b) runs. Color indicates the source amplitude of the events in $\mathrm{dB}$.

Fig.4 shows six examples of the vertical temperature profiles $T(z)$ obtained during unloading at different times. As can be seen the heating and cooling processes are not homogeneous on the sample as a consequence of the inhomogeneous growth and shrinkage of the austenic regions as well as the unavoidable heat difussion process. A clear picture of the dynamics of the inhomogeneous heating (during loading) and cooling (during unloading) of the sample can be obtained by plotting the evolution of the temperature profiles as a function of time in a color map, as shown in Fig.5 (a) and (b). Note that only the zone imaged by the IR camera is monitored. In these plots, the progression of the phase transition fronts, where latent heat is exchanged, corresponds to the sharp knees in the isotherms, associated with the moving heat sources and heat sinks.

By a proper numerical post-processing of the thermal profile it is possible to obtain the dynamics and location of the heat power source and sink densities. ${ }^{12,13,18}$. Considering that the problem is essentially one dimensional, and neglecting heat losses towards the air, one can describe the heat trans- port problem by considering the Fourier equation which can be expressed as:

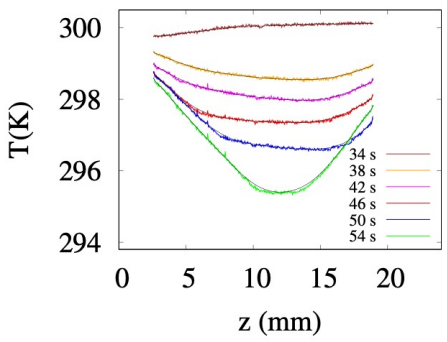

FIG. 4. Examples of vertical temperature profiles for selected times (as indicated by the labels) during unloading. The thin black lines indicate the corresponding smoothed averages used for the computation of the power source and sink density.

$$
\Sigma(z, t) \equiv \frac{\sigma(z, t)}{C \rho}=\frac{\partial T(z, t)}{\partial t}-\frac{\kappa}{C \rho} \frac{\partial^{2} T(z, t)}{\partial z^{2}}
$$

where $C$ is the specific heat, $\rho$ is the density, $\kappa$ is the thermal conductivity and $\sigma$ is the heat production term. Positive values for $\Sigma$ indicate power source density and negative values indicate power sink density.

By multiplying Eq.(1) by the frame discretization $\Delta t$, one can obtain an expression for the numerical computation of the power source and sink density as:

$$
\Sigma^{*}=\frac{\partial T}{\partial k}-\kappa^{*} \frac{\partial^{2} T}{\partial i^{2}}
$$

where $\Sigma^{*}=\frac{\sigma \Delta t}{C \rho}, \kappa^{*}=\frac{\kappa \Delta t}{C \rho \Delta x^{2}}$ and the first derivative refers to changes of temperature per frame and the second derivative to second order changes of temperature per square pixel. Prior to the computation of the numerical derivatives, the $T(i, k)$ profiles have been smoothed by averaging over 5 frames (from $k-2$ to $k+2)$ in the time direction and 101 pixels in the spatial direction (from $i-50$ to $i+50$ ). Examples of the smoothed profiles are shown in Fig. 4 with black lines on top of the original $T(i, k)$ profiles. The second numerical step is to compute the first order time derivatives and the second order spatial derivatives using 5 point formulas, considering increments of 20 frames and 30 pixels respectively.

Using $\rho=7.12 \mathrm{~g} \mathrm{~cm}^{-3}, C=0.45 \mathrm{~J} \mathrm{~K}^{-1} \mathrm{~g}^{-1}$ and $\kappa=45 \mathrm{~W}$ $\mathrm{m}^{-1} \mathrm{~K}^{-1}$ for $\mathrm{Cu}-\mathrm{Al}-\mathrm{Ni}$ alloys ${ }^{19}$ we have computed the local distribution and time dependence of $\Sigma$. Results are shown in Figure 5 (c) and (d). Numerical errors, near the upper and lower edges of the plots, are due to the bias of the smoothing algorithm due to the boundaries of the available data.

The thermal source (sink) map nicely coincides with the mechanical energy map derived from AE experiments. It is to be noticed, however, that for the loading run, the AE trace displays a clear curvature that is not observed in the IR data. 

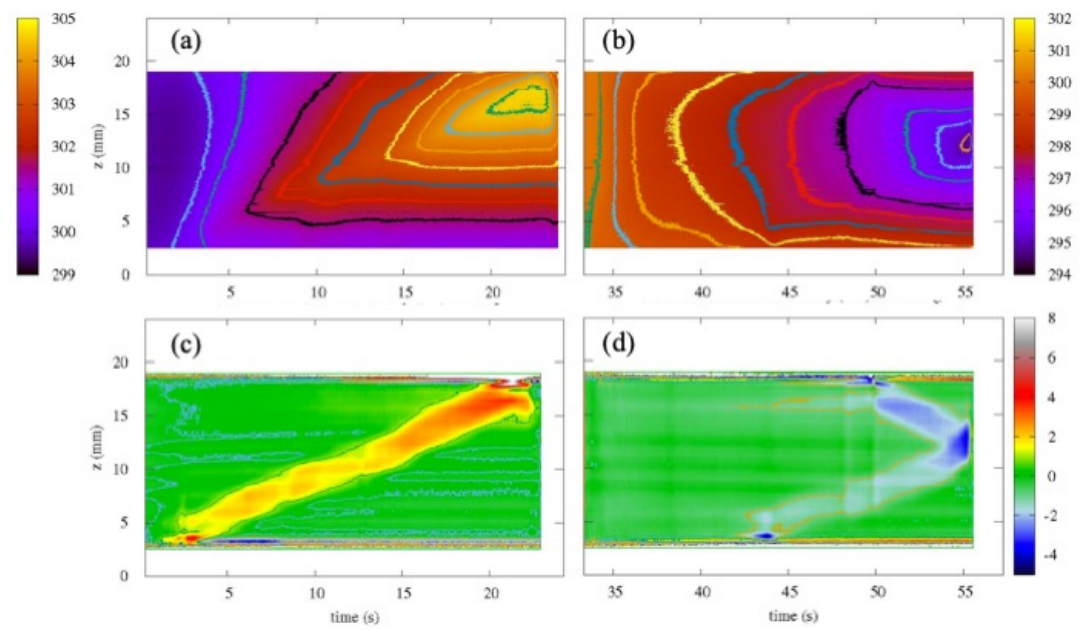

FIG. 5. Top panels: Evolution of the vertical temperature profile (in K) as a function of time during loading (a) and unloading (b). Contour lines correspond to $0.5 \mathrm{~K}$ separation. Bottom panels: power source density (c) and power sink density (b) (in $\mathrm{Ks}^{-1}$ ) as a function of time during loading and unloading respectively. Contour lines coorrespond to $0 \mathrm{Ks}^{-1}$ and $1 \mathrm{Ks}^{-1}$ for loading and $0 \mathrm{Ks}^{-1}$ and $-1 \mathrm{Ks}^{-1}$ for unloading.

This deviation (that does not occur in the unloading process) can be understood since the location of the AE events has been performed assuming uniform sound velocity in the sample. It is known that the austenitic and martensitic phase have different sound propagation speeds. In general, this error is difficult to correct because it would depend on the particular geometry of the phase fronts, but it is expected that the error would be smaller when several fronts are present simultaneoulsy in the sample, which is what happens during the unloading regime. It is also worth noting that the dynamic behaviour of the power source and sink densities is also coincident with the dynamics of the AE data. An interesting point to remark is the fact that the merging of two fronts (as occurs at the end of the unloading run) gives rise to a local power sink which is significantly larger than the standard power sink during the norma displacement of an individual front.

A global view of the coincidence between thermal and acoustic effects can be gained by integrating $\Sigma$ along the whole sample volume in order to compute the evolution of the total power source and sink. Results are shown in Fig. 2 as red (source) and blue (sink) lines. There is a good correlation between the two set of experiments, and this spatially averaged correlation is similar to the results derived from high sensitivity calorimetry and $\mathrm{AE}$ count rate techniques ${ }^{14}$. An added value to the computation of heat sources and sinks is that integration over the whole duration of the loading (and unloading) processes renders the total exchanged heat in the imaged region which is related to the latent heat of the transformation. In our experiments we have obtained $0.59 \mathrm{~J}$ for loading and $-0.42 \mathrm{~J}$ for unloading. these values should be comapred with the theoretically available latent heat in the imaged re- gion which is $0.48 \mathrm{~J}$. The agreement is rather good given our one dimensional approximation, the numerical uncertainties in the computation, and the fact that heat transport to air and through the grips has not been considered. The fact that for loading the estimation is larger than the value expected from latent heat could be due to heat transport from the upper part of the sample that is not imaged by the IR camera.

Recently Acoustic Emission sources have been correlated with sudden changes in the strain fields (avalanches) measured by an optical grid method ${ }^{6}$. Here we have extended that study by resolving the dynamics of the heat sources and sinks which have been found to be well correlated with the sources of mechanical energy. The combined results of these independent techniques showing good correlation between thermal and mechanical events, may help in understanding the dynamics of martensitic transition and its interaction with disorder and sample boundary conditions. In particular, we have revealed phenomena such as domain nucleation, and front growth through front propagation, affected by pinning and jamming.

It is finally worth mentionning that the connection between heat exchange with specific details in the martensitic microstructure (enhanced heat absorption at the merging of martensitic interfaces) may provide useful information in the potential use of shape memory alloys for elastocaloric cooling. Materials with tailored microstructures, and some control of disorder distribution, can be designed which promote interface collisions at specific sites which may enable, for instance, localized cooling at desired positions.

The data that support the findings of this study are available from the corresponding author upon reasonable request. 


\section{ACKNOWLEDGMENTS}

Financial support is acknowledged to the Spanish Ministry of Economy and Competitiveness, Project No. MAT201675823-R, and to the Independent Research Fund Denmark (contract 8022-00277B). M.R. acknowledges a Juan de la Cierva Fellowship.

${ }^{1}$ K. Otsuka and C.M. Wayman, Shape Memory Materials, Cambridge University Press, 1998

${ }^{2}$ J.M. Jani, M. Leary, A. Subic, M.A. Gibson, Mater and Design 56, 1078 (2014)

${ }^{3}$ F.J.Perez-Reche, C.Triguero, G.Zanzotto and L.Truskinovsky, Phys. Rev. B 94, 144102 (2016)

${ }^{4}$ Z.Y.Wei, W.Sun, Q.Shen, Y.Shen, Y.F.Zhang, E.K.Liu and J.Liu, Appl. Phys. Lett. 114, 101903 (2019)

${ }^{5}$ X.Balandraud, N.Barrera, P.Biscari, M.Grédiac and G.Zanzotto, Phys. Rev. B 91, 174111 (2015)

${ }^{6}$ B.Blaysat, X.Balandraud, M.Grédiac, E.Vives, N.Barrera and G.Zanzotto, Commun Mater 1, 3 (2020)

${ }^{7}$ A. Planes, L. Mañosa, E. Vives, J. Alloys and Compounds 577S, S699

(2013). $\quad{ }^{8}$ E. Vives, J. Ortín, L. Mañosa, I. Ràfols, R. Pérez-Magrané, A. Planes, Phys.

Rev. Lett. 72, 1694 (1994)
${ }^{9}$ E.Vives, D.Soto-Parra, L.Mañosa, R.Romero, and A.Planes, Phys. Rev. B 84, 060101(R) (2011)

${ }^{10}$ J.A. Shaw and S. Kyriakidesm, Acta Mater. 45, 683 (1997).

${ }^{11}$ H.Ossmer, F.Lambrecht, M.Gultig, C.Chubla, E. Quandt and M.Kohl, Acta Materialia 81, 9-20 (2014).

${ }^{12} \mathrm{D}$. Delpueyo, M. Grédiac, X. Balandraud and C. Badulescu, Mechanics of Materials 45, 34-51 (2012).

${ }^{13}$ C.Bubulinca, X.Balandraud, D.Delpueyo, M.Grédiac, S.Stanciu, ${ }^{13}$ C.Bubulinca, X.Balandraud, D.Delpueyo, M.Grédiac, S.Stanciu,
M.Abrudeanu, A.G.Paiasu, M.M.Dicu and E.L.Nitu, Journal of Optoelectronics and Advanced Materials 15, 544-549 (2013).

${ }^{14}$ A. Planes, J.L. Macqueron, M. Morin, G. Guenin, phys. stat. sol. (a) 66 , 717.

${ }^{15}$ J. Tusek, K. Engelbrecht, D. Eriksen, S. Dall'Olio, J. Tusek, N. Pryds, Nature Energy 1, 16134 (2016)

${ }^{16}$ E.Bonnot, R.Romero, X.Illa, L.Mañosa, A.Planes and E.Vives, Phys. Rev. B 76, 064105 (2007),

${ }^{17}$ E. Bonnot, E.Vives, L.Mañosa, A.Planes and R.Romero, Phys. Rev. B 78, 094104 (2008).

${ }^{18}$ C.Beitone, X.Balandraud, M.Grédiac, D.Delpueyo, C.Tilmant and F.Chausse, in Residual Stress, Thermomechanics \& Infrared Imaging, Hybrid Techniques and Inverse Problems, Volume 9, Ed. by S.Bossuyt, G.Schajer and A. Carpinteri, Conference Proceedings of the Society for Experimental Mechanics Series. Springer, Cham (2016).

${ }^{19}$ I.Ruiz-Larrea, A.López-Echarri, E.H.Bocanegra, M.L.Nó and J.M.San Juan Materials Science and Engineering: A 438-440, 779-781 (2006). 

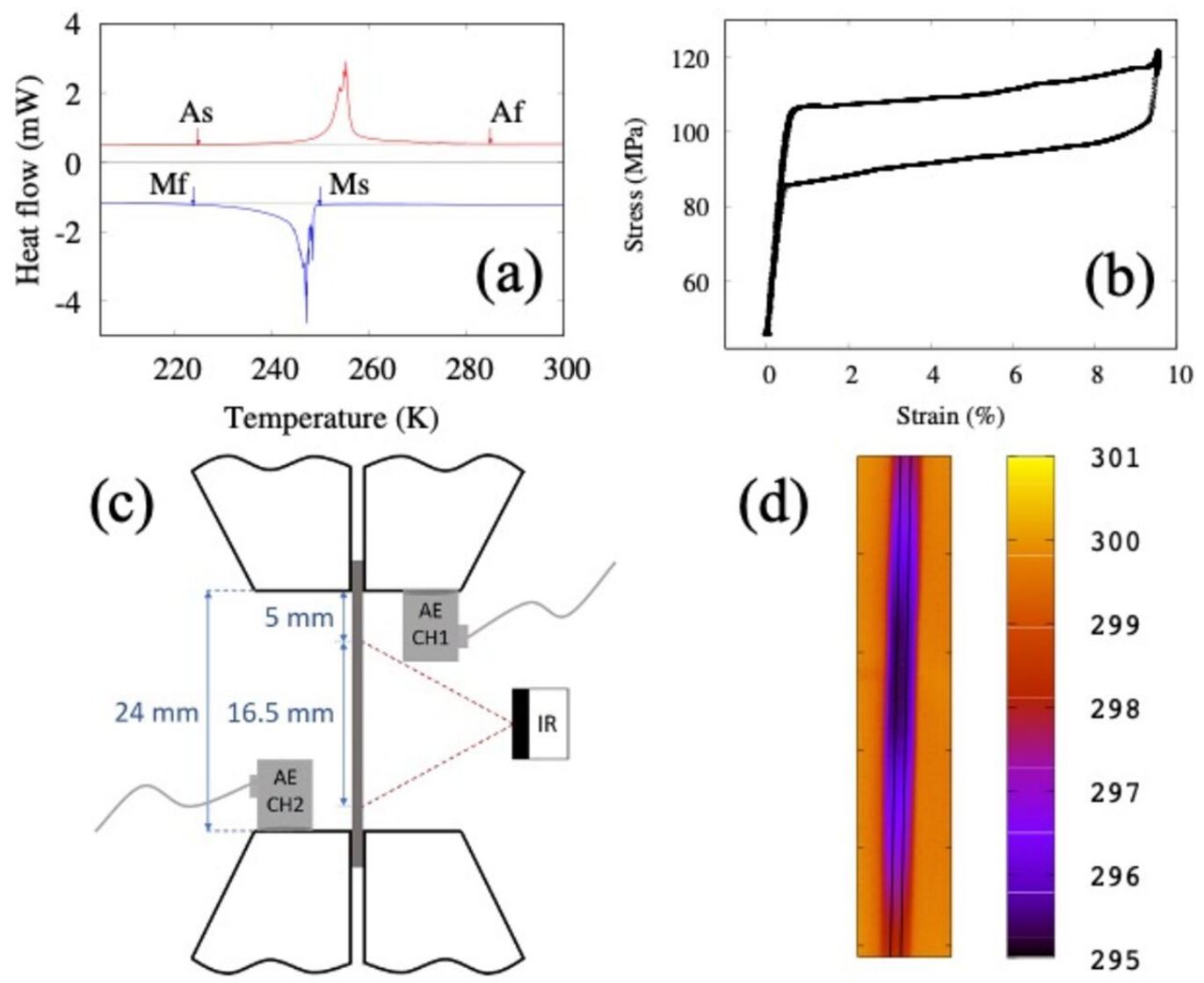







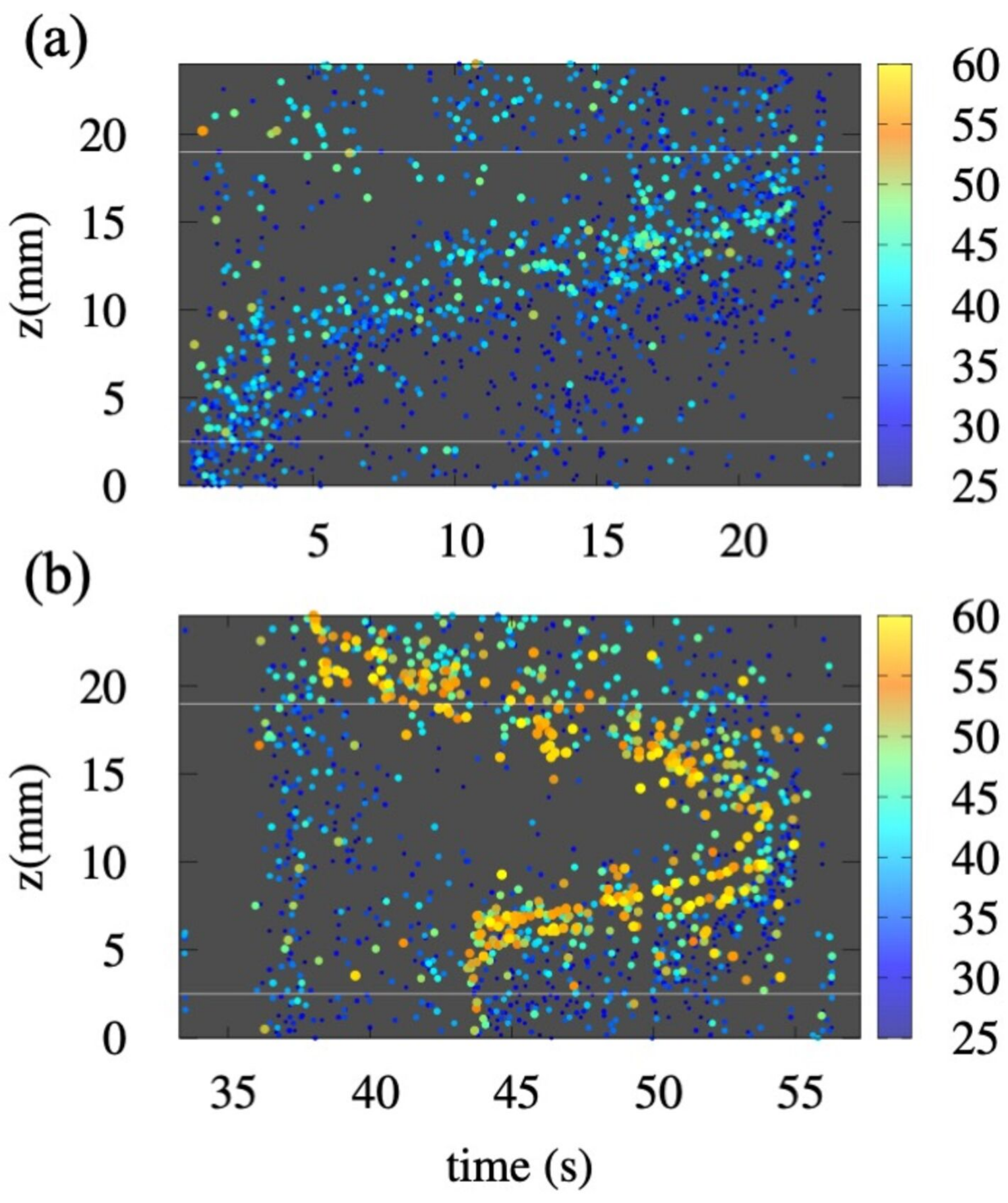




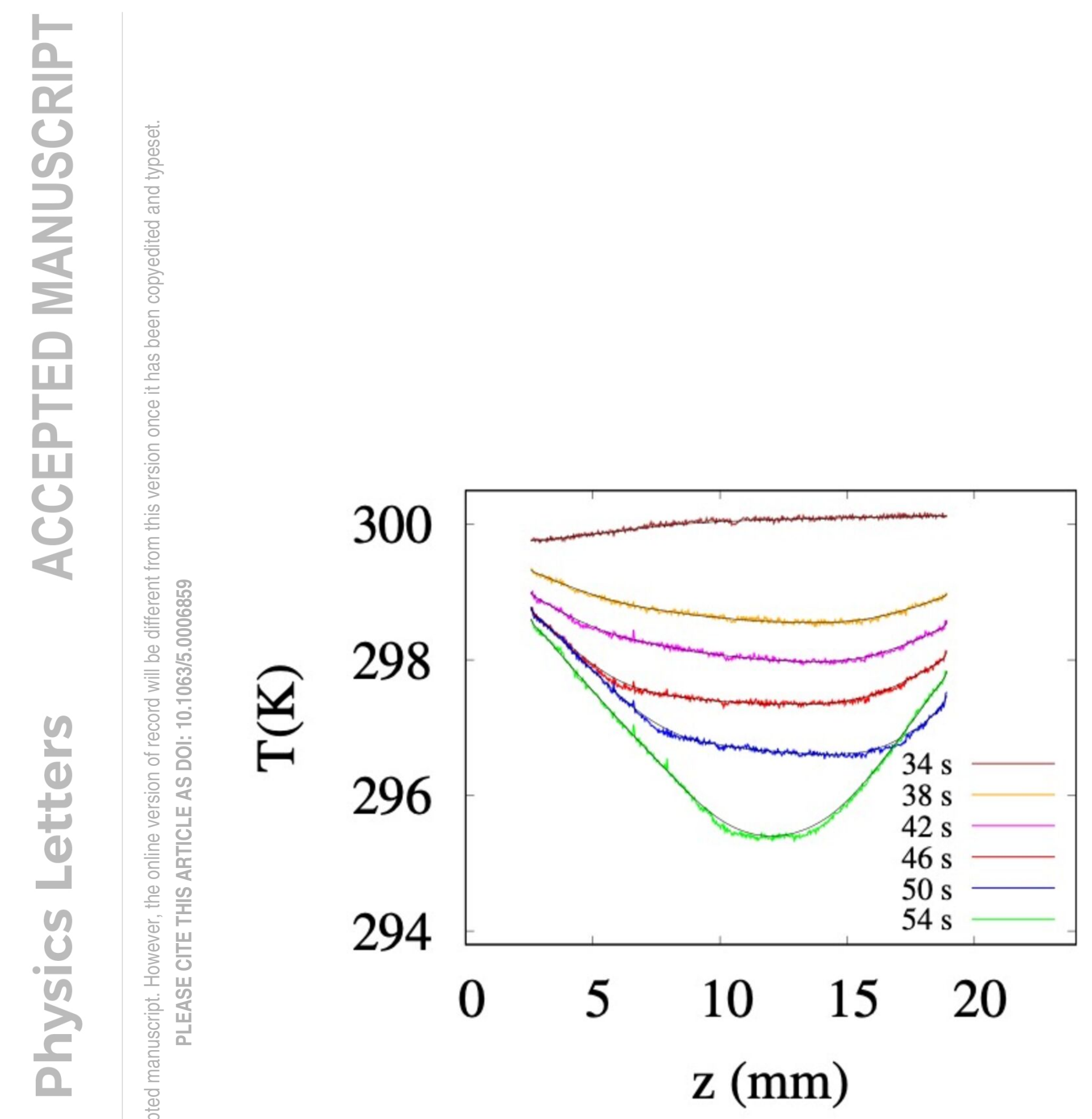

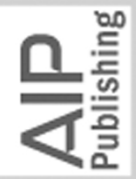




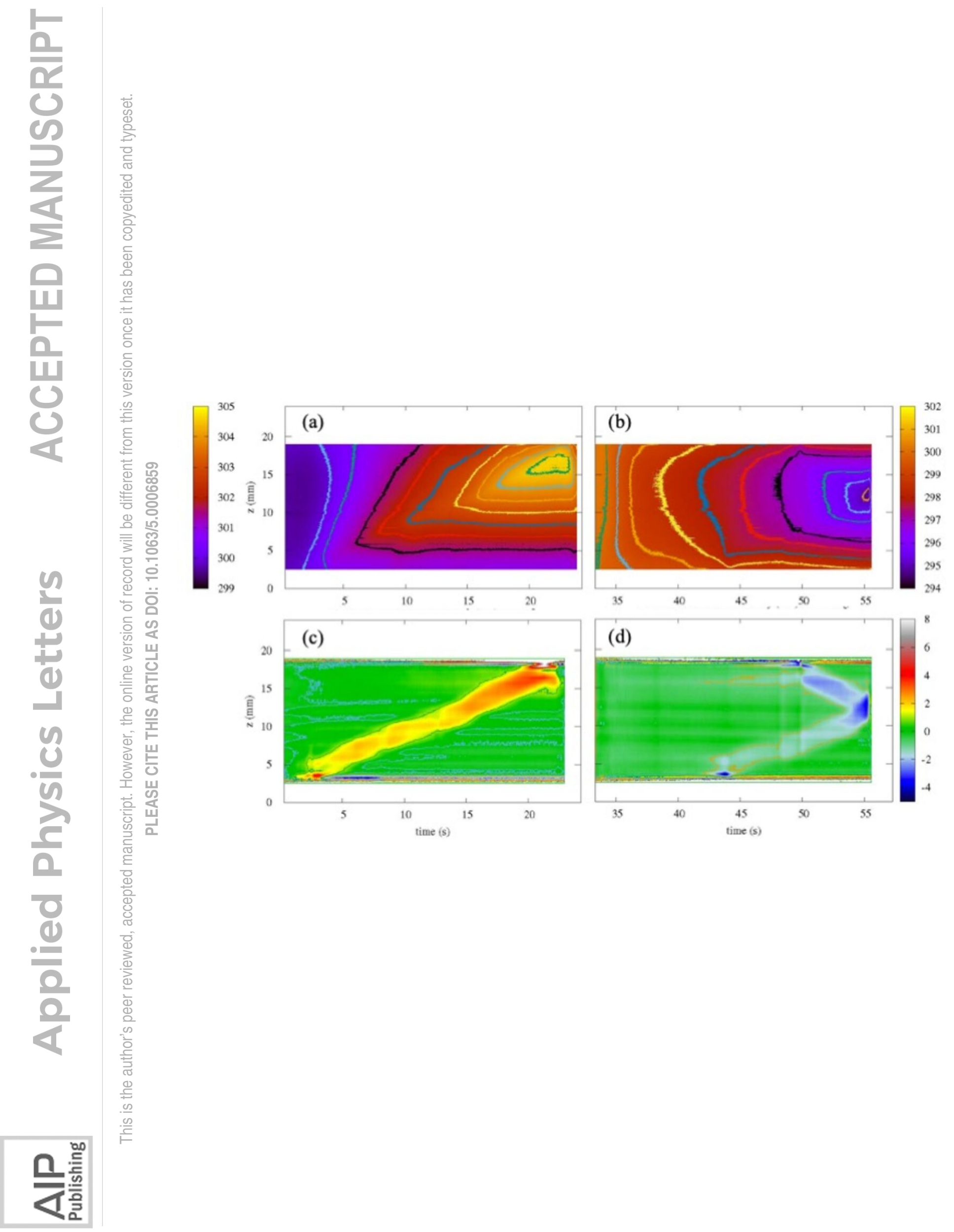

